\title{
Reduction of miR-132-3p contributes to gastric cancer proliferation by targeting MUC13
}

\author{
LIANG HE $^{1}$, LINLIN QU ${ }^{2}$, LIJING WEI ${ }^{2}$, YAN CHEN ${ }^{1}$ and JIAN SUO ${ }^{1}$ \\ Departments of ${ }^{1}$ Gastrointestinal Surgery and ${ }^{2}$ Laboratory Medicine, \\ The First Hospital of Jilin University, Changchun, Jilin 130021, P.R. China
}

Received September 30, 2015; Accepted January 16, 2017

DOI: $10.3892 / \mathrm{mmr} .2017 .6347$

\begin{abstract}
Abnormal expression of epidermal growth factor receptor (EGFR) signaling and microRNAs (miRNAs) has been widely seen in gastric cancer. The present study focused on the miRNAs that regulate human epidermal growth factor receptor (HER) activation through mucin 13 (MUC13). The protein level of MUC13 was demonstrated to be significantly increased in gastric cancer tissues compared with normal tissues by western blot analysis and immunohistochemistry. TargetScan bioinformatic predictions indicated that miRNA (miR)-212-3p and miR-132-3p may bind to the 3'-untranslated region of MUC13. Further investigation revealed that miR-132-3p was significantly decreased in gastric cancer tissues compared with normal tissues, whereas miR-212-3p expression was unaffected. Luciferase assays and western blot confirmed MUC13 as a target gene of miR-132-3p. Inhibition of miR-132-3p enhanced gastric cancer cell migration through activation of HER2, extracellular signal-regulated kinase (ERK) and Akt serine/threonine kinase (Akt) signaling, which was a similar effect to that of MUC13 overexpression. In summary, reduction of miR-132-3p may contribute to gastric cancer proliferation by targeting MUC13.
\end{abstract}

\section{Introduction}

Gastric cancer is one of the most common malignant tumors worldwide, causing 738,000 deaths in 2008 (1). Major contributory factors to gastric cancer include Helicobacter pylori infection, diet, alcoholic consumption and smoking $(2,3)$.

Receptor tyrosine kinase (RTK) pathways have key roles in the progression of various tumors (4-6), with aberrant epidermal growth factor receptor (EGFR) signaling demonstrated to be particularly common (7); EGF family proteins

Correspondence to: Dr Jian Suo, Department of Gastrointestinal Surgery, The First Hospital of Jilin University, 71 Xinmin Street, Changchun, Jilin 130021, P.R. China

E-mail: suojian150930@163.com

Key words: miR-132-3p, gastric cancer, cell migration, MUC13, HER2 have been revealed to be significantly overexpressed in $>60 \%$ of tumors $(8,9)$. The main EGF family members include EGFR [also termed human epidermal growth factor receptor 1(HER1)], HER2 (also termed ErbB2), HER3 (also termed ErbB3) and HER4 (also termed ErbB4). EGFR and HER2 are often significantly upregulated in gastric cancer, and are considered to be well-established oncogenes (10). Mucin 13 (MUC13) has also been demonstrated to be aberrantly upregulated in various tumors (11-13). Exogenous expression of MUC13 contributes to abnormal cell proliferation, motility and tumor growth (13), and overexpression of MUC13 results in the activation of HER2, extracellular signal-regulated kinase (ERK) and Akt serine/threonine kinase (Akt), and the reduction of p53 expression (12). However, few studies have, thus far, investigated the expression of MUC13 in gastric cancer.

MicroRNAs (miRNAs) are small non-coding RNAs that widely control gene expression at the post-transcriptional level (14-16). Due to the oncogenic or tumor suppressive roles of miRNAs, abnormal expression can lead to the initiation, formation and progression of tumors. For example, numerous miRNAs are differentially expressed in gastric cancer, including miRNA-199a-3p (miR-199a-3p), miR-429 and miR-34a (14-16). The present study aimed to investigate miRNAs that regulate the expression of MUC13 in gastric cancer.

\section{Materials and methods}

Patient selection and biopsy collection. In the present study, biopsies were taken from both tumor tissue and the adjacent normal tissue of 40 patients receiving adenocarcinoma surgery of the stomach or esophageal junction. Samples were collected from July 2012 to November 2014 and written consent was provided by all individuals. The collection of biopsies was approved by the Ethics Committee of the First Hospital of Jilin University (Changchun, China) in accordance with the Declaration of Helsinki. All biopsy samples were reviewed by an experienced pathologist to validate the diagnosis.

Immunohistochemistry. Paraffin-embedded tissues fixed in $4 \%$ buffered paraformaldehyde were cut into $5 \mu \mathrm{m}$ sections and washed three times ( 5 min per wash) in phosphate-buffered saline (PBS), then incubated in $3 \% \mathrm{H}_{2} \mathrm{O}_{2}$ for $30 \mathrm{~min}$ at room temperature. Sections were blocked by incubation 
with $10 \%$ goat serum in PBS (Origene Technologies, Inc., Rockville, MD, USA) for $30 \mathrm{~min}$ at $37^{\circ} \mathrm{C}$, then incubated with the MUC13 primary antibody (1:80; catalog no. ab124654; Abcam, Cambridge, UK) for $24 \mathrm{~h}$ at $4^{\circ} \mathrm{C}$. Sections were washed with PBS, then incubated with secondary antibody (biotin-labelled goat anti-mouse immunoglobulin G; 1:200; catalog no. SP-9000D; Origene Technologies, Inc.) for $1 \mathrm{~h}$ at $4^{\circ} \mathrm{C}$. Following washing with PBS, sections were incubated with horseradish peroxidase conjugated streptavidin (1:200) for $1 \mathrm{~h}$ at room temperature, then with diaminobenzidine $/ \mathrm{H}_{2} \mathrm{O}_{2}$ for $15 \mathrm{~min}$ at room temperature. Following dehydration in gradient alcohol, and transparentizing in xylene, sections were mounted with glycerol and observed under a microscope. In control sections, the primary antibody was replaced with $1 \%$ calf serum (Origene Technologies, Inc.).

Cell culture. Gastric cancer cell line, MKN28 was purchased from American Type Culture Collection (ATCC, Manassas, VA, USA) and cultured in RPMI-1640 (GE Healthcare Life Sciences, Logan, UT, USA) supplemented with $10 \%$ fetal bovine serum (FBS; Invitrogen; Thermo Fisher Scientific, Inc., Waltham, MA, USA), streptomycin (100 mg/ml) and penicillin $(100 \mathrm{U} / \mathrm{ml})$ at $37^{\circ} \mathrm{C}$ in a humidified atmosphere containing $5 \% \mathrm{CO}_{2}$. Recent reports have suggested that the MKN28 gastric carcinoma cell line used in this study is contaminated with another gastric carcinoma cell line, MKN74 (17).

RNA extraction. Total RNA was extracted from gastric tissues or MKN28 cells using TRIzol reagent according to the manufacturers' instructions (Thermo Fisher Scientific, Inc., Waltham, MA, USA).

Reverse transcription-quantitative polymerase chain reaction $(R T-q P C R)$. Total RNA was reverse transcribed using Takara MicroRNA Reverse Transcription Kit (Takara Bio, Inc., Otsu, Japan) with specific primers for miR-132-3p (GTCGTATCCAGTGCAGGGTCCGAGGTATTCGCACT GGATACGACCGACC) and U6 (GTCGTATCCAGTGCAG GGTCCGAGGTATTCGCACTGGATACGACAAATAT).

Subsequently, the PCR amplification was performed. $1 \mathrm{mg}$ of cDNA was used for qPCR using SYBR green Master mix (Roche Diagnostics, Basel, Switzerland) on a Roche Lightcycler 480 (Roche Diagnostics) at $95^{\circ} \mathrm{C}$ for $10 \mathrm{~min}$ followed by 50 cycles of $95^{\circ} \mathrm{C}$ for $10 \mathrm{sec}, 55^{\circ} \mathrm{C}$ for $10 \mathrm{sec}, 72^{\circ} \mathrm{C}$ for $5 \mathrm{sec} ; 99^{\circ} \mathrm{C}$ for $1 \mathrm{sec} ; 59^{\circ} \mathrm{C}$ for $15 \mathrm{sec} ; 95^{\circ} \mathrm{C}$ for $1 \mathrm{sec}$; then cooling to $40^{\circ} \mathrm{C}$. Relative miRNA expression of miR-132-3p was normalized against the endogenous control, U6, using the $\Delta-\Delta \mathrm{Cq}$ method (18).

Transient transfection. A total of $6 \times 10^{5}$ cells were equally seeded in the 6-well plates with $2 \mathrm{ml}$ RPMI-1640 culture medium containing serum and antibiotics. At the same time, miR-132-3p mimic, inhibitor, miR negative control or siRNA targeting MUC13 (CCAGCUUGUUGAGGUAGAAGUAGU A) or non-target control siRNA (Shanghai GenePharma Co., Ltd., Shanghai, China) were mixed with HiperFect transfection reagent (Qiagen $\mathrm{GmbH}$, Hilden, Germany) and incubated at room temperature for $10 \mathrm{~min}$. The complex was then transfected into MKN28 cells for $48 \mathrm{~h}$.
Cell viability analysis. To examine cell viability, MKN28 cells were seeded in 96-well plates at a density of $1.0 \times 10^{4}$ cells/per well. miR-132 mimics, inhibitors or a scramble/non-targeting oligo negative control (NC) were transfected into cells at 24 , 48, $72 \mathrm{~h}$ after seeding of cells. MTT assay was performed as previously described (19).

Adenovirus vector construction and transfection. The adenovirus vector (Ad)-MUC13 and Ad-control (Ad-con) were purchased from the Chinese National Human Genome Center (Beijing, China). In brief, $6 \times 10^{5}$ cells were equally seeded in the 6-well plates with $2 \mathrm{ml}$ RPMI-1640 culture medium containing serum and antibiotics. Following $24 \mathrm{~h}$, the cells were transfected with Ad-MUC13 or Ad-con at the density of 100 multiplicity of infection (MOI) for $48 \mathrm{~h}$. The transfection efficiency was calculated as the green fluorescent protein-positive cells/all cells in each field x100\%.

Luciferase target assay. The 3'untranslated region (UTR) of MUC13 containing the predicted target site for miR-20a-5p, was cloned into the pmirGLO (Promega Corporation, Madison, WI, USA) luciferase reporter vector which had been cleaved at the SacI and XhoI sites. Details of PCR procedures are described as follows: a heated initial denaturation step at $95^{\circ} \mathrm{C}$ for $10 \mathrm{~min}$, followed by 40 cycles at $95^{\circ} \mathrm{C}$ for $15 \mathrm{sec}$, $55^{\circ} \mathrm{C}$ for $45 \mathrm{sec}$ and $72^{\circ} \mathrm{C}$ for $30 \mathrm{sec}$. Prior to conducting the luciferase reporter assay, $5 \times 10^{4}$ cells per well were seeded in 24-well plates in a $500 \mu \mathrm{l}$ medium and cultured for $18 \mathrm{~h}$. The cells were transfected with the modified firefly luciferase vector $(500 \mathrm{ng} / \mu \mathrm{l})$ mixed with Vigofect transfection reagent, according to the manufacturer's protocol. Following a $48 \mathrm{~h}$ continuous exposure, the luciferase activities from firefly and renilla were measured with the Dual-luciferase reporter assay system (Promega Corporation). Renilla activity was used as the normalized parameter.

Establishment of MUC13-expressing MKN28 stable cell line. MKN28 cells were transfected with pmirGLO-MUC13-3'UTR or empty vector (pmirGLO) using VigoFect transfection reagent (Vigorus Biotechnology, Beijing, China). Individual G418 resistant clones ( $1 \mathrm{mg} / \mathrm{ml}$; Invitrogen; Thermo Fisher Scientific, Inc.) were selected and applied for further study.

Western blotting analyses. Tissue or MKN28 cell protein was extracted using RIPA buffer (Solarbio Science \& Technology Co., Ltd., Beijing, China). A bicinchoninic protein assay kit (Pierce; Thermo Fisher Scientific, Inc.) was used to determine the protein concentration. Equal quantities of protein $(15 \mu \mathrm{g})$ were resolved by $10 \%$ SDS-PAGE and transferred onto a PVDF membrane. The protein was detected with primary antibodies, MUC13 (catalog no. ab124654; Abcam), HER 2 (catalog no. 4290; 1:1,000), p-ERK (catalog no. 1150; 1:1,000), ERK (catalog no. 9194; 1:1,000), p-Akt (catalog no. 8200; 1:1,000), Akt (catalog no. 9840; 1:1,000) and GAPDH (catalog no. 2118; 1:5,000) all obtained from Cell Signaling Technology, Inc., (Danvers, MA, USA) overnight at $4^{\circ} \mathrm{C}$. Nonspecific binding was blocked using $8 \%(\mathrm{w} / \mathrm{v})$ milk in Tris-buffered saline with 1\% Tween-20 (TBST; Beijing SolarBio Science \& Technology Co., Ltd.) for $2 \mathrm{~h}$ 
at room temperature. Following several washes with TBST, the membranes were incubated with horseradish-peroxidase (HRP)-conjugated goat anti-rabbit and anti-mouse IgG or HRP-conjugated mouse anti-goat IgG (all 1:5,000; Origene Technologies, Inc.) for $2 \mathrm{~h}$ at room temperature and then washed. GAPDH was used as the internal control. Signals were detected with enhanced chemiluminescence according to the manufacturer's protocol (EMD Millipore, Billerica, MA, USA). ImageJ software (National Institutes of Health, Bethesda, MD, USA) was used for density analysis.

Bioinformatic predictions. To determine the potential miRNAs that target MUC13, bioinformatic prediction was performed using TargetScan (http://www.targetscan.org).

Cell invasion assay. Invasion of cells were examined using a Transwell system (Invitrogen; Thermo Fisher Scientific, Inc.). The MKN28 cells transfected with miR-132 inhibitors or ad-MUC13 were cultured in the lower chamber with fresh medium containing $10 \%$ FBS. After incubation for $24 \mathrm{~h}$ at $37^{\circ} \mathrm{C}$, the cells on the upper chamber was stained with $0.5 \%$ crystal violet and dissolved in $10 \%$ acetic acid for measurement of absorbance at $560 \mathrm{~nm}$.

Cell migration assay. The in vitro wound healing assay was performed as previously described (20). Briefly, MKN28 cells were seeded in 6-well plates to form a confluent monolayer. The monolayer was scratched with a sterile $10 \mu \mathrm{l}$ pipette tip, and the floating cells were carefully removed by washing with PBS. Then, the cells were cultured in RPMI-1640 medium without $\mathrm{FBS}$ at $37^{\circ} \mathrm{C}$ in a $5 \% \mathrm{CO}_{2}$ atmosphere. The wound scratches were photographed at 0 and $12 \mathrm{~h}$ then scraped to collect cells.

Statistical analysis. The data are expressed as the mean \pm standard error. The number of independent experiments was represented by 'n'. Data were analyzed using SPSS software, version 13.0 (SPSS, Inc., Chicago, IL, USA). P<0.05 was considered to indicate a statistically significant difference.

\section{Results}

Upregulation of MUC13 in gastric cancer tissues. Initially, the expression of MUC13 in gastric cancer tissues was examined. Western blot analysis demonstrated that MUC13 was significantly upregulated in gastric cancer tissues compared with adjacent normal tissues (Fig. 1A). Immunohistochemistry analysis also demonstrated the enhanced expression of MUC13 in gastric cancer tissues (Fig. 1B).

MUC13 is a target gene of miR-132-3p in gastric cancer. To identify the potential miRNAs that regulate the expression of MUC13, the TargetScan online prediction program was used. As demonstrated in Fig. 2A, two putative conserved binding miRNAs, miR-132-3p and miR-212-3p, were identified to potentially bind the 3'untranslated region (3'UTR) of MUC13. The present study determined that miR-132-3p levels were reduced in gastric cancer tissues compared with adjacent normal control tissue, whereas miR-212-3p did not demonstrate significant a change (Fig. 2B). Subsequently, the

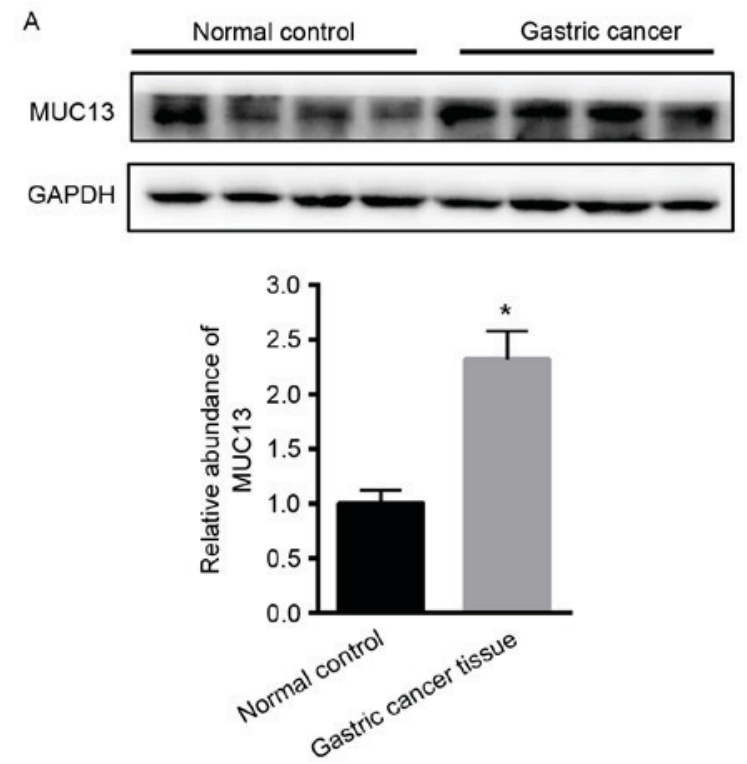

B

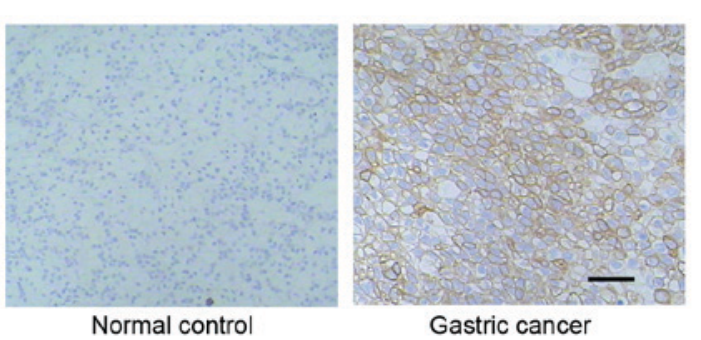

Figure 1. Expression of MUC13 was increased in gastric cancer tissues compared with normal tissues. (A) Western blot analysis. ${ }^{*} \mathrm{P}<0.05$ vs. control. (B) Immunohistochemistry analysis. $\mathrm{n}=5$ independent tissues. MUC13, mucin 13 .

3'UTR of MUC13 was cloned into the pmirGLO plasmid. A dual luciferase reporter assay demonstrated that miR-132-3p significantly decreased the relative luciferase units of pmirGLO-MUC13-3'UTR compared with with the empty vector pmirGLO (Fig. 2C). Additionally, miR-132-3p mimics or inhibitors were transfected into MKN28 cells. RT-qPCR analysis revealed that transfection with miR-132-3p mimics markedly enhanced the level of miR-132-3p, whereas transfection with miR-132-3p inhibitors significantly reduced the level of miR-132-3p (Fig. 2D). Overexpression of miR-132-3p significantly increased the level of miR-132-3p, however decreased the protein level of MUC13 (Fig. 2E). By contrast, inhibition of miR-132-3p reduced the level of miR-132-3p, however increased the expression of MUC13 (Fig. 2E). These data indicated that reduction of miR-132-3p led to enhanced MUC13 expression in gastric cancer cells.

Overexpression of MUC13 increases the activation of HER signaling and cell invasion and migration in MKN28 cells. To explore the role of MUC13 on gastric cancer progression, cell invasion and migration were analyzed. As demonstrated in Fig. 3A, the transfection efficiency of Ad-MUC13 or Ad-con was nearly $100 \%$ (Fig. 3A). No alterations of MUC13 protein levels were observed following simple transfection of blank adenovirus vectors (data not shown). A Transwell assay demonstrated that overexpression of MUC13 significantly 
A

Human MUC13 3'UTR

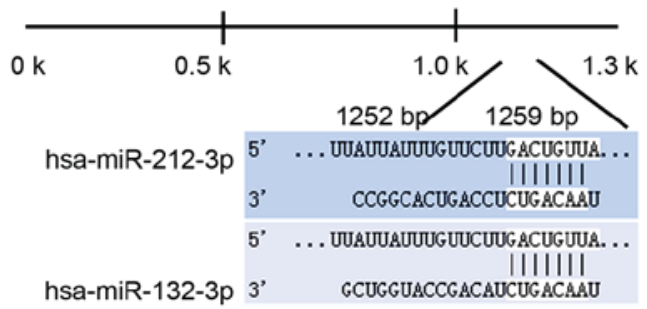

C

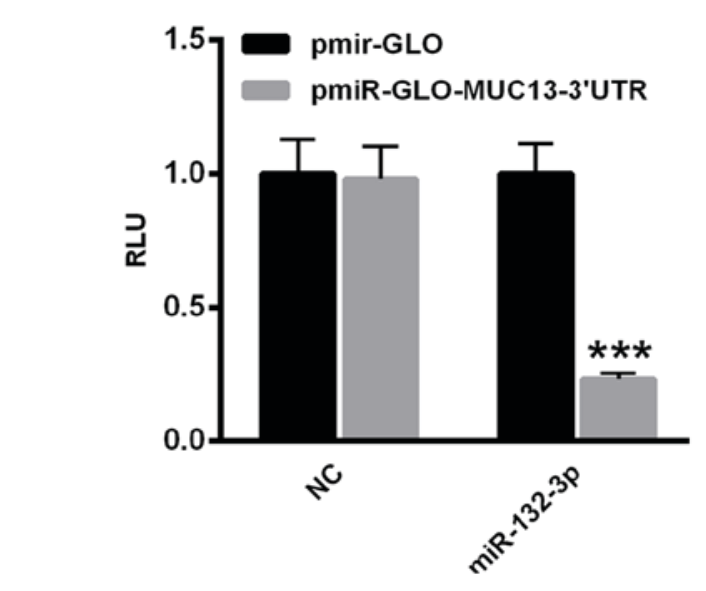

B
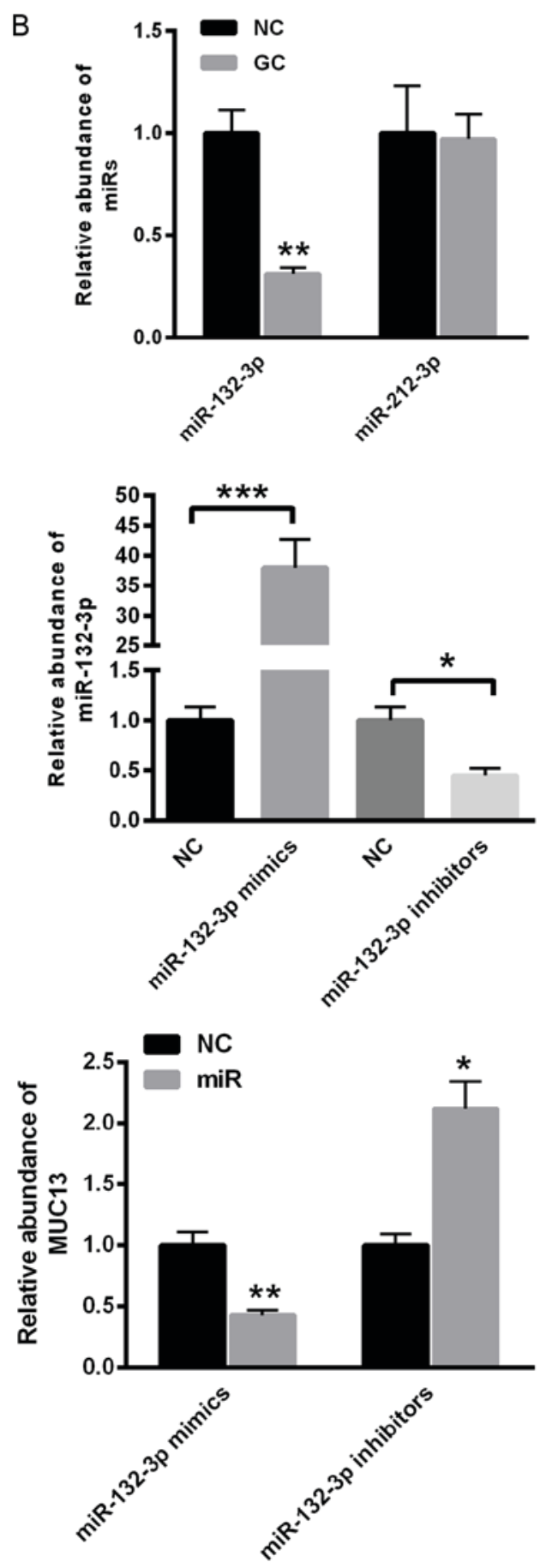

Figure 2. Reduction of miR-132-3p led to enhanced MUC13 expression in gastric cancer cells. (A) TargetScan prediction programs indicated that miR-132-3p and miR-212-3p may bind to the 3'UTR of MUC13. (B) Reverse transcription-quantitative polymerase chain reaction was performed to determine the level of miR-132-3p and miR-212-3p in gastric cancer tissues (GC) ( $\mathrm{n}=5$ independent samples) and normal control (NC). (C) Luciferase assay indicated that miR-132-3p decreased the relative luciferase activity of pmirGLO-MUC13-3'UTR. (D) RT-qPCR analysis of miR-132-3p levels following transfection of miR-132-3p mimics or inhibitors. (E) Transfection of miR-132-3p mimics significantly decreased the protein level of MUC13 in MKN28, and transfection of miR-132-3p inhibitors significantly decreased the protein level of MUC13. ${ }^{*} \mathrm{P}<0.05,{ }^{* *} \mathrm{P}<0.01,{ }^{* * * *} \mathrm{P}<0.001$ vs. control. MUC13, mucin 13; UTR, untranslated region; miR, microRNA; RLU, relative luciferase unit; NC, negative control.

enhanced cell invasion capacity (Fig. 3B). Furthermore, a scratch assay demonstrated that MKN28 cell migration was enhanced by MUC13 overexpression (Fig. 3C). The signaling downstream of MUC13 was also examined. As demonstrated in Fig. 3D, overexpression of MUC13 obviously enhanced the level of HER2, and the phosphorylation of ERK and Akt (Fig. 3D).
Knockdown of MUC13 partially reverses miR-132-3p inhibition-induced MKN28 cell invasion and migration. To explore whether miR-132-3p exerts its role through MUC13, miR-132-3p inhibitors were transfected into MKN28 cells. As demonstrated in Fig. 4A, inhibition of miR-132-3p significantly enhanced the protein level of MUC13, and enhanced the level of HER2, and ERK and Akt phosphorylation. Notably, an 
A
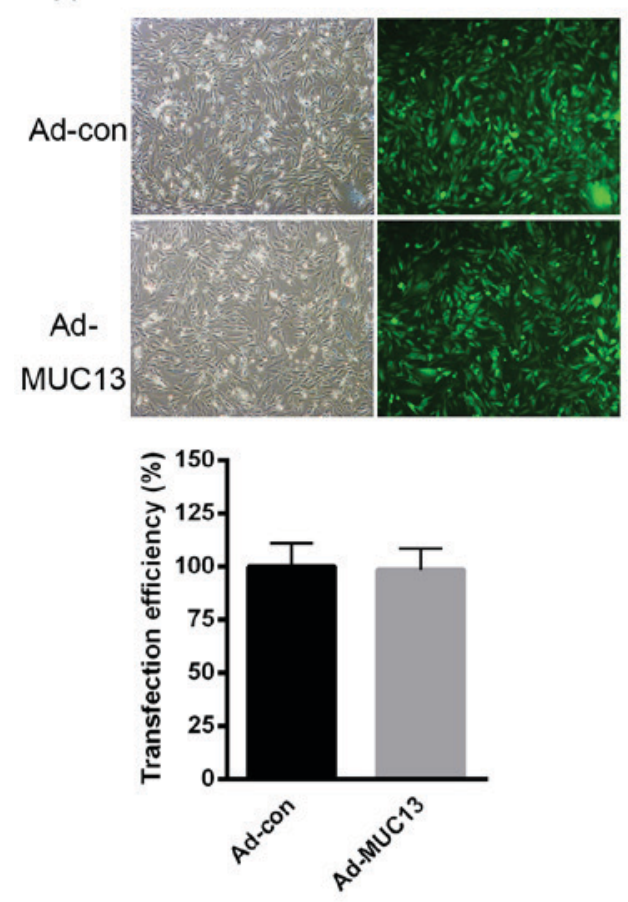

C

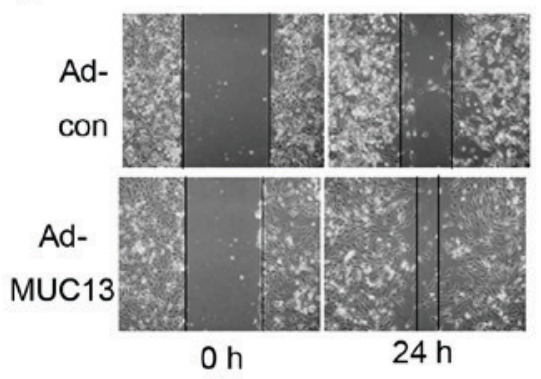

B
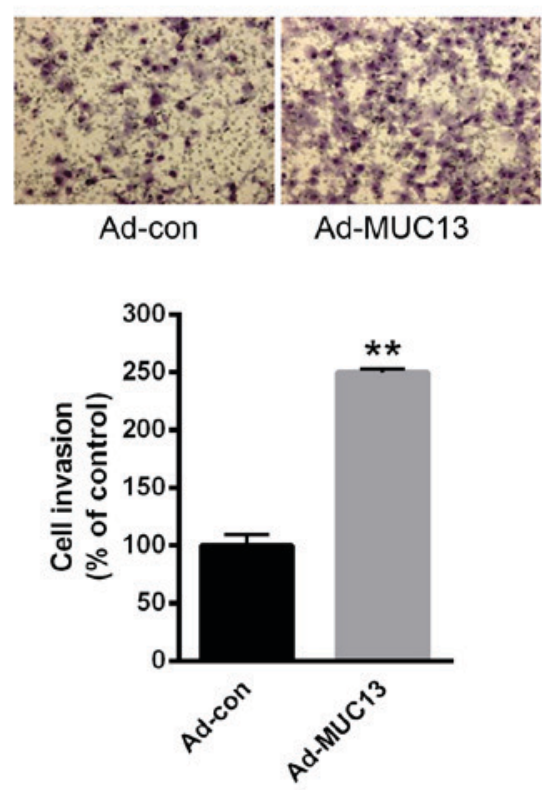

D

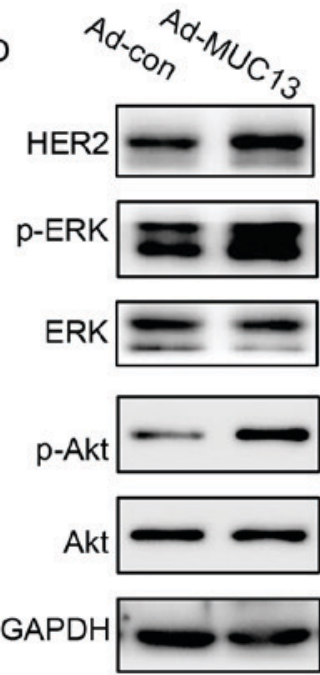

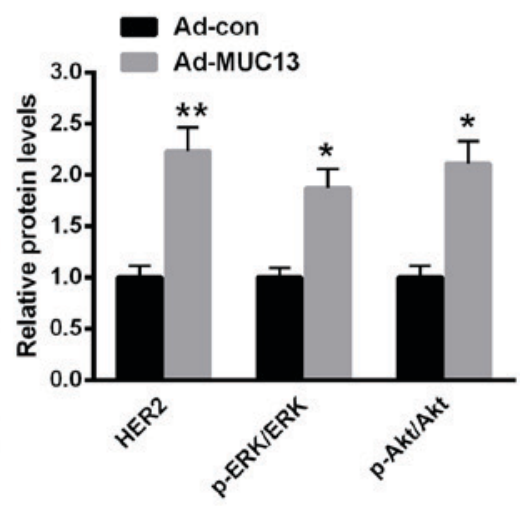

Figure 3. Overexpression of MUC13 prompted MKN28 cell invasion and migration through activation of HER signaling. (A) Transfection efficiency of Ad-MUC13 or Ad-con. (B) Transwell assay demonstrated that overexpression of MUC13 significantly enhanced MKN28 cell invasion capacity. (C) Scratch assay demonstrated that MKN28 cell migration was significantly enhanced when MUC13 was overexpressed. (D) Overexpression of MUC13 obviously enhanced the expression of HER2, and the phosphorylation of ERK and Akt. $\mathrm{n}=3$ independent experiments, ${ }^{*} \mathrm{P}<0.05,{ }^{* * *} \mathrm{P}<0.01$ vs. control. Ad, adenovirus; con, control; MUC13, mucin 13; HER2, human epidermal growth factor receptor 2; p-, phosphorylated; ERK, extracellular signal-regulated kinase; Akt, Akt serine/threonine kinase.

siRNA targeting MUC13 was selected to suppress the expression of MUC13. miR-132-3p inhibition reduced the effects of MUC13 siRNA on HER2 expression, and ERK and Akt activation (Fig. 4B). Additionally, the effect was on cell invasion and migration was also determined. miR-132-3p inhibitors reduced the effect of MUC13 knockdown on cell invasion and migration (Fig. 4C and D).

\section{Discussion}

Mucins are considered as potential oncogenes and possible therapeutic targets in various malignancies (21-23). As a high-molecular-weight transmembrane glycoprotein, MUC13 is reported to be frequently overexpressed in various epithelial carcinomas, including gastric, colorectal and ovarian cancers (24). MUC13 includes three EGF-like domains and a cytoplasmic domain with phosphorylation sites, which trigger the activation of HER2 signaling (24). The current study examined MUC13 expression in gastric cancer tissues and detected that MUC13 protein levels were significantly increased compared with adjacent normal tissues.

A previous study reported that overexpression of MUC13 significantly enhanced the activation of HER2, ERK and Akt (13). To validate the role of MUC13 in gastric cancer 
A
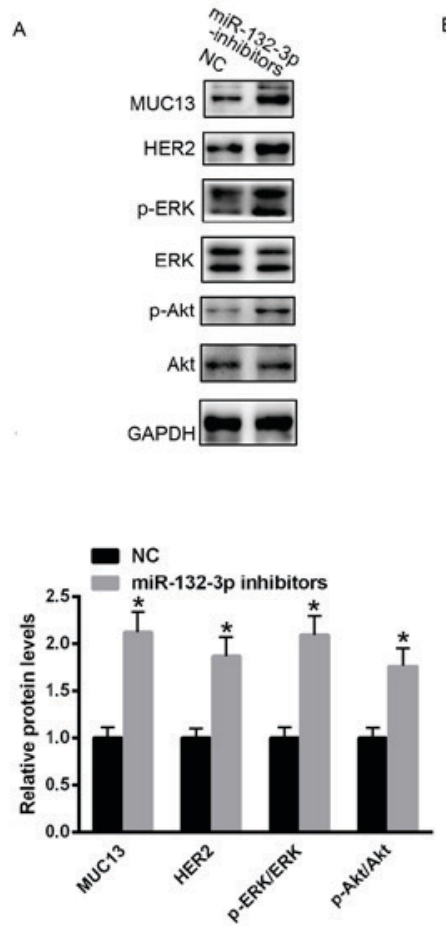

B

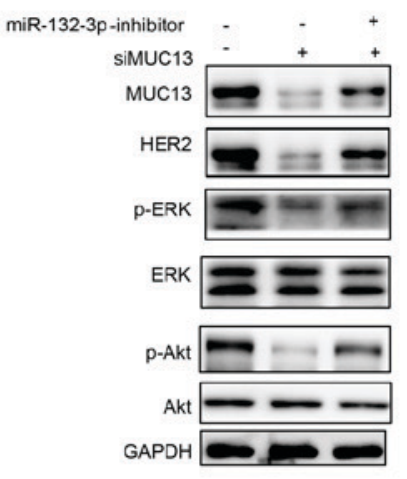

NC

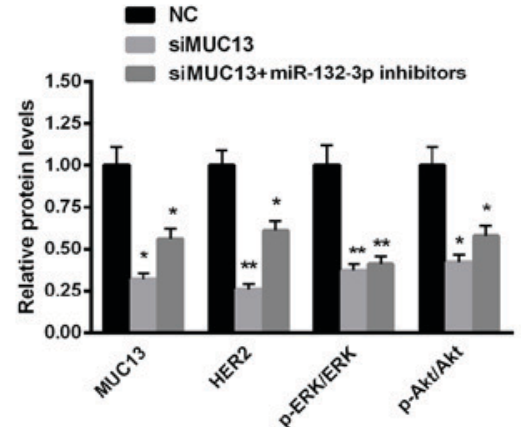

c

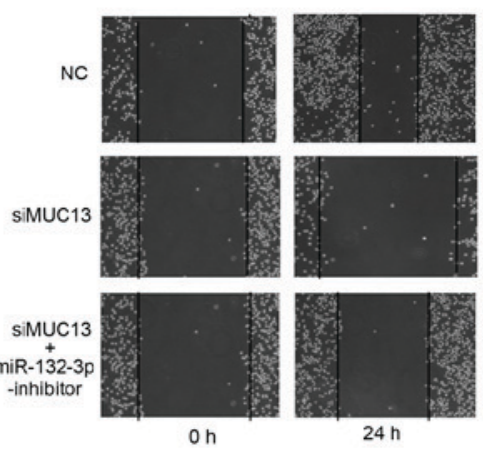

D

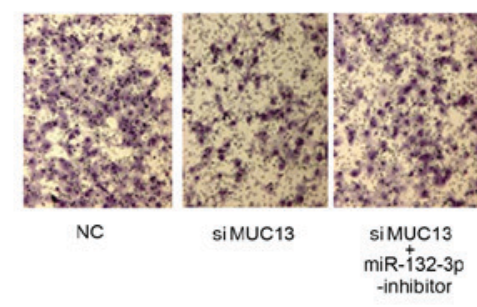

Figure 4. Knockdown of MUC13 partially reverses miR-132-3p inhibition-induced effects on MKN28 cell invasion and migration. (A) Inhibition of miR-132-3p significantly enhanced the expression of MUC13 and the activation of downstream signaling. (B) Knockdown of MUC13 reversed the effects of miR-132-3p inhibition on HER2, ERK and Akt. (C) Cell invasion and (D) migration were monitored in cells transfected with miR-132-3p inhibitors or/and si-MUC13 or NC. $n=3$ independent experiments, ${ }^{*} \mathrm{P}<0.05,{ }^{* *} \mathrm{P}<0.01$ vs. control. NC, negative control; miR, microRNA; MUC13, mucin 13; HER2, human epidermal growth factor receptor 2; p-, phosphorylated; ERK, extracellular signal-regulated kinase; Akt, Akt serine/threonine kinase; siMUC13, MUC13-targeting small interfering RNA.

progression, MUC13 was exogenously expressed in MKN28 cells. Overexpression of MUC13 observably enhanced gastric cancer cell invasion and migration. By contrast, knockdown of MUC13 decreased MKN28 cell invasion and migration. Due to the three EGF domains, MUC13 is considered to stabilize the protein level of EGF receptors, particularly HER2, thus enhancing the activation of ERK and Akt. Activation of phosphoinositide 3-kinase/Akt and mitogen-activated protein kinase signaling through HER 2 enhances tumorigenesis in various types of cancer $(25,26)$.

miRNAs are increasingly reported to be differentially expressed in various tumors either as oncogenes or tumor suppressors (27-29). In a previous study, miR-145 was identified to target MUC13 inhibiting the tumor growth and invasion in pancreatic tissues. The present study aimed to elucidate novel miRNAs that regulate the expression of MUC13 in gastric cancer (12). Bioinformatic predictions suggested that miR-132-3p and miR-212-3p may bind to the 3'UTR of MUC13. A luciferase reporter assay and western blot analysis demonstrated that MUC13 is the target gene of miR-132-3p. Further study demonstrated that miR-132-3p was obviously decreased in gastric cancer tissues compared with normal adjacent tissues. In line with MUC13 overexpression, reduced miR-132-3p contributed to gastric cancer cell invasion and migration. Notably, inhibition of miR-132-3p reduced the activation of ERK and Akt even in cells transfected the specific siRNA targeting MUC13, suggesting the tumor suppressor role of miR-132-3p in gastric cancer through MUC13.
To conclude, miR-132-3p may function as a tumor suppressor in gastric cancer tissues by targeting MUC13 and subsequently prompting the activation of HER2 signaling.

\section{References}

1. Jemal A, Bray F, Center MM, Ferlay J, Ward E and Forman D: Global cancer statistics. CA Cancer J Clin 61: 69-90, 2011.

2. Kato M and Asaka M: Recent knowledge of the relationship between Helicobacter pylori and gastric cancer and recent progress of gastroendoscopic diagnosis and treatment for gastric cancer. Jpn J Clin Oncol 40: 828-837, 2010.

3. Li L, Ying XJ, Sun TT, Yi K, Tian HL, Sun R, Tian JH and Yang KH: Overview of methodological quality of systematic reviews about gastric cancer risk and protective factors. Asian Pac J Cancer Prev 13: 2069-2079, 2012.

4. Deng Z, Ma S, Zhou H, Zang A, Fang Y, Li T, Shi H, Liu M, Du M, Taylor PR, et al: Tyrosine phosphatase SHP-2 mediates C-type lectin receptor-induced activation of the kinase Syk and anti-fungal TH17 responses. Nat Immunol 16: 642-652, 2015.

5. Chung C: Tyrosine kinase inhibitors for epidermal growth factor receptor gene mutation-positive non-small cell lung cancers: An update for recent advances in therapeutics. J Oncol Pharm Pract 22: 461-476, 2016

6. Shabani M, Naseri J and Shokri F: Receptor tyrosine kinase-like orphan receptor 1: A novel target for cancer immunotherapy. Expert Opin Ther Targets 19: 941-955, 2015.

7. Hatanpaa KJ, Burma S,Zhao D and Habib AA: Epidermal growth factor receptor in glioma: Signal transduction, neuropathology, imaging, and radioresistance. Neoplasia 12: 675-684, 2010.

8. Mellinghoff IK, Wang MY, Vivanco I, Haas-Kogan DA, Zhu S, Dia EQ, Lu KV, Yoshimoto K, Huang JH, Chute DJ, et al: Molecular determinants of the response of glioblastomas to EGFR kinase inhibitors. N Engl J Med 353: 2012-2024, 2005.

9. Mizukami T, Togashi Y, Sogabe S, Banno E, Terashima M, De Velasco MA, Sakai K, Fujita Y, Tomida S, Nakajima TE, et al: EGFR and HER2 signals play a salvage role in MEK1-mutated gastric cancer after MEK inhibition. Int J Oncol 47: 499-505, 2015. 
10. Yk W, Cf G, T Y, Z C, Xw Z, Xx L, Nl M and Wz Z: Assessment of ERBB2 and EGFR gene amplification and protein expression in gastric carcinoma by immunohistochemistry and fluorescence in situ hybridization. Mol Cytogenet 4: 14, 2011.

11. Liu C, Smet A, Blaecher C, Flahou B, Ducatelle R, Linden S and Haesebrouck F: Gastric de novo Muc13 expression and spasmolytic polypeptide-expressing metaplasia during Helicobacter heilmannii infection. Infect Immun 82: 3227-3239, 2014.

12. Khan S, Ebeling MC, Zaman MS, Sikander M, Yallapu MM, Chauhan N, Yacoubian AM, Behrman SW, Zafar N, Kumar D, et al: MicroRNA-145 targets MUC13 and suppresses growth and invasion of pancreatic cancer. Oncotarget 5 7599-7609, 2014

13. Chauhan SC, Ebeling MC, Maher DM, Koch MD, Watanabe A Aburatani H, Lio Y and Jaggi M: MUC13 mucin augments pancreatic tumorigenesis. Mol Cancer Ther 11: 24-33, 2012.

14. Liu G, Jiang C, Li D, Wang R and Wang W: MiRNA-34a inhibits EGFR-signaling-dependent MMP7 activation in gastric cancer. Tumour Biol 35: 9801-9806, 2014.

15. Liu D, Xia P, Diao D, Cheng Y, Zhang H, Yuan D, Huang C and Dang C: MiRNA-429 suppresses the growth of gastric cancer cells in vitro. J Biomed Res 26: 389-393, 2012.

16. Li C, Li JF, Cai Q, Qiu QQ, Yan M, Liu BY and Zhu ZG: miRNA-199a-3p in plasma as a potential diagnostic biomarker for gastric cancer. Ann Surg Oncol 20 (Suppl 3): S397-S405, 2013.

17. Nardone RM: Curbing rampant cross-contamination and misidentification of cell lines. Biotechniques 45: 221-227, 2008.

18. Livak KJ and Schmittgen TD: Analysis of relative gene expression data using real-time quantitative PCR and the 2(-Delta Delta C(T)) Method. Methods 25: 402-408, 2001.

19. Yang F, Wang H, Jiang Z, Hu A, Chu L, Sun Y and Han J: MicroRNA19a mediates gastric carcinoma cell proliferation through the activation of nuclear factor-kB. Mol Med Rep 12: 5780-5786, 2015.
20. Liang CC, Park AY and Guan JL: In vitro scratch assay: A convenient and inexpensive method for analysis of cell migration in vitro. Nat Protoc 2: 329-333, 2007.

21. Khan S, Ansarullah, Kumar D, Jaggi M and Chauhan SC: Targeting microRNAs in pancreatic cancer: Microplayers in the big game. Cancer Res 73: 6541-6547, 2013.

22. Luo H, Guo W, Wang F, You Y, Wang J, Chen X, Wang J, Wang Y, Du Y, Chen X, et al: miR-1291 targets mucin 1 inhibiting cell proliferation and invasion to promote cell apoptosis in esophageal squamous cell carcinoma. Oncol Rep 34: 2665-2673, 2015.

23. Wakata K, Tsuchiya T, Tomoshige K, Takagi K, Yamasaki N, Matsumoto K, Miyazaki T, Nanashima A, Whitsett JA, Maeda Y and Nagayasu T: A favourable prognostic marker for EGFR mutant non-small cell lung cancer: Immunohistochemical analysis of MUC5B. BMJ Open 5: e008366, 2015.

24. Maher DM, Gupta BK, Nagata S, Jaggi M and Chauhan SC: Mucin 13: Structure, function, and potential roles in cancer pathogenesis. Mol Cancer Res 9: 531-537, 2011.

25. Arias-Romero LE and Chernoff J: p21-activated kinases in Erbb2-positive breast cancer: A new therapeutic target? Small GTPases 1: 124-128, 2010.

26. Moasser MM: The oncogene HER2: Its signaling and transforming functions and its role in human cancer pathogenesis. Oncogene 26: 6469-6487, 2007.

27. Han HS, Son SM, Yun J, Jo YN and Lee OJ: MicroRNA-29a suppresses the growth, migration, and invasion of lung adenocarcinoma cells by targeting carcinoembryonic antigen-related cell adhesion molecule 6. FEBS Lett 588: 3744-3750, 2014.

28. Sun S, Sun P, Wang C and Sun T: Downregulation of microRNA-155 accelerates cell growth and invasion by targeting c-myc in human gastric carcinoma cells. Oncol Rep 32: 951-956, 2014.

29. Xia Y and Gao Y: MicroRNA-181b promotes ovarian cancer cell growth and invasion by targeting LATS2. Biochem Biophys Res Commun 447: 446-451, 2014. 